\title{
A Universal Law for Cell Uptake of One- Dimensional Nanomaterials
}

ARTICLE in NANO LETTERS · JANUARY 2014

Impact Factor: $13.59 \cdot$ DOI: 10.1021/nl404727m · Source: PubMed

CITATIONS

13
DOWNLOADS

94
VIEWS

133

3 AUTHORS:

Xin Yi

Brown University

20 PUBLICATIONS 310 CITATIONS

SEE PROFILE

Huajian Gao

Brown University

529 PUBLICATIONS 19,113 CITATIONS

SEE PROFILE
Xinghua Shi

Chinese Academy of Sciences

42 PUBLICATIONS 595 CITATIONS

SEE PROFILE 


\title{
A Universal Law for Cell Uptake of One-Dimensional Nanomaterials
}

\author{
Xin $\mathrm{Yi}^{\dagger}{ }^{\dagger}$ Xinghua Shi, ${ }^{\ddagger}$ and Huajian $\mathrm{Gao}^{*}{ }^{\dagger}$ \\ ${ }^{\dagger}$ School of Engineering, Brown University, Providence, Rhode Island 02912, United States \\ ${ }^{\ddagger}$ LNM, Institute of Mechanics, Chinese Academy of Sciences, Beijing 100190, China
}

ABSTRACT: Understanding cell interaction with one-dimensional nanomaterials, including nanotubes, nanowires, nanofibers, filamentous bacteria, and certain nanoparticle chains, has fundamental importance to many applications such as biomedical diagnostics, therapeutics, and nanotoxicity. Here we show that cell uptake of one-dimensional nanomaterials via receptor-mediated endocytosis is dominated by a single dimensionless parameter that scales with the membrane

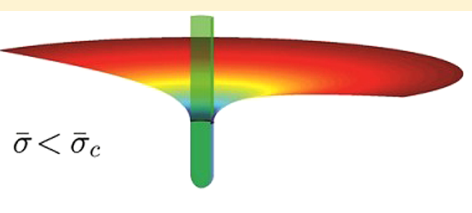

$\bar{\sigma}>\bar{\sigma}_{c}$

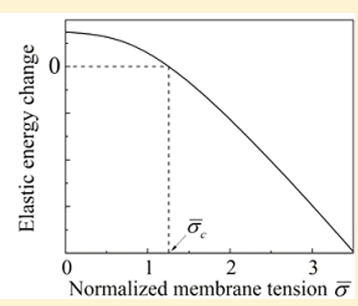
the membrane bending stiffness. It is shown that as cell membrane internalizes one-dimensional nanomaterials the uptake follows a near-perpendicular entry mode at small membrane tension but it switches to a near-parallel interaction mode at large membrane tension.

KEYWORDS: Cell membrane, nanotubes, cell interaction, microtubule, filopodia, molecular dynamics
$\mathrm{U}$ nderstanding interaction mechanisms between nanomaterials and cell membranes is essential for biomedical diagnostics, therapeutics, and nanotoxicity. Although extensive studies have been conducted on cellular uptake of (spherical) nanoparticles, relatively little is known about how cells interact with one-dimensional (1D) nanomaterials with radius at the nanoscale but length at a much larger scale, such as nanotubes and filamentous bacteria. ${ }^{1-3}$ Recent experiments showed that carbon nanotubes undergo endocytic entry into liver and mesothelial cells through tip recognition and then rotation to a near-perpendicular entry mode. ${ }^{1}$ Similar tip entry phenomena were also observed in phagocytosis of high-aspect ratio filamentous Escherichia coli bacteria. ${ }^{3}$ In another type of experiment, it was found that nanoparticles adhering to a substrate-supported flat phospholipid vesicle spontaneously form particle-chains encapsulated inside lipid tubes that protrude out of the vesicle at low membrane tension, but when the membrane tension is increased by adding $\mathrm{Ca}^{2+}$ to enhance adhesion between the vesicle and substrate, the lipid tubes retract back to the vesicle. ${ }^{4}$ Here we show that these seemingly different phenomena can all be understood with a unified theoretical model of an elastic fluid membrane interacting with a $1 \mathrm{D}$ nanomaterial.

The above experiments suggest that there exist two basic modes of interaction between cells and $1 \mathrm{D}$ nanomaterials: tip recognition followed by near-perpendicular entry versus nearparallel adhering on the membrane. However, the underlying mechanisms of different modes of interaction have not been elucidated. In this Letter, we present the first theoretical model on cellular uptake of $1 \mathrm{D}$ nanomaterials, which accurately accounts for the elastic deformation energy associated with the wrapping of an initially flat membrane around a nanotube oriented at different orientations with respect to the membrane. First, we note that a lipid membrane, subjected to various types of loading, deforms and equilibrates immediately on a time scale of less than one second. ${ }^{5-7}$ By contrast, cell uptake of nanomaterials with a time scale from hundreds of seconds to tens of minutes ${ }^{8-10}$ can usually be regarded as a process limited by diffusion of receptors in the cell membrane toward the contact region. ${ }^{1,11}$ For simplicity, here we do not consider the dynamic process of receptor diffusion in the membrane; rather we focus on a relatively short time window around a given point of time in which the total area of the contact region remains constant while the nanotube adjusts its entry angle to reduce the energy of the system. It will be shown that membrane tension, membrane bending stiffness, and tube radius collectively determine the uptake mode through a single dimensionless parameter.

Without loss of generality, we consider an initially flat membrane wrapping around a semi-infinite cylindrical nanotube with a hemispherical end-cap (Figure 1). The total energy of the system is expressed in terms of Canham-Helfrich functional as ${ }^{12,13}$

$$
E_{\mathrm{tot}}(\theta)=E_{\mathrm{b}}+\sigma \Delta A-\gamma A_{\mathrm{c}}
$$

where $E_{\mathrm{b}}=\int 2 \kappa H^{2} d A$ is the bending energy, $\kappa$ being the bending rigidity of the membrane, $H$ is the local mean curvature, and $d A$ is the membrane surface element; $\sigma$ is the membrane tension and $\Delta A$ the excess surface area induced by wrapping; $\gamma$ is the specific adhesion energy and $A_{c}$ is the contact area. The bending energy associated with the Gaussian curvature remains constant for the problem and is neglected. Spontaneous curvature is not considered here. All length scales

Received: December 20, 2013

Revised: January 13, 2014

Published: January 23, 2014 


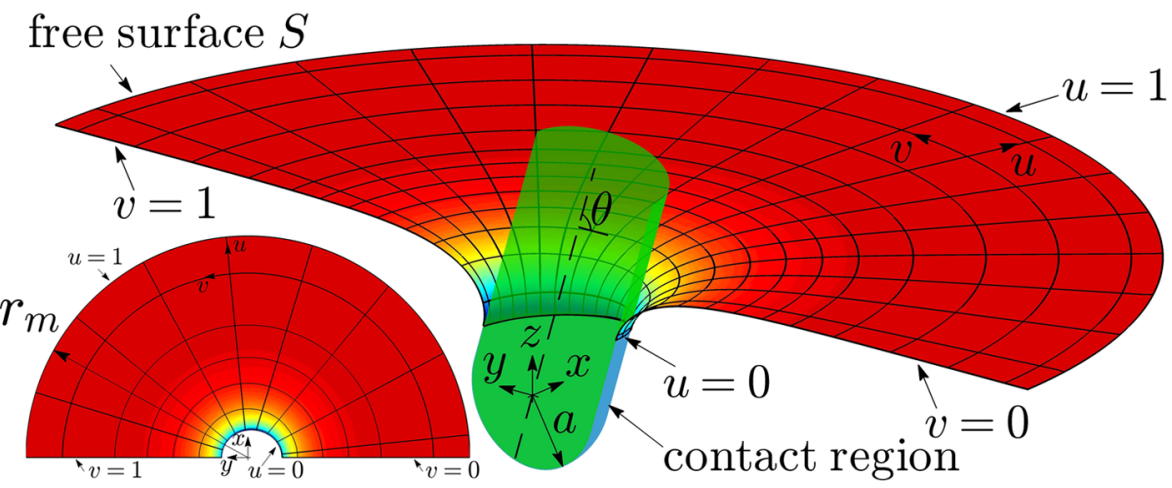

Figure 1. Schematic of an initially flat membrane wrapping around a nanotube (green). A nanotube of radius $a$ is seen to enter membrane at an angle $\theta$. Because of limited rate of receptor diffusion, the total area of the contact region (blue) is assumed to be fixed in the analysis while the nanotube can adjust the entry angle $\theta$ to reduce the system energy. Curvilinear coordinates $u, v=0,1$ define the boundaries of the free part $S$ of the membrane, and colors on $S$ denote the relative height of the membrane. The hemispherical cap of the nanotube is bounded by $x^{2}+y^{2}+z^{2}=a^{2}$ and $z \sin \theta-y$ $\cos \theta=0$ in the adopted Cartesian coordinate $x y z$. (Lower left corner) Top view of the free part $S$.

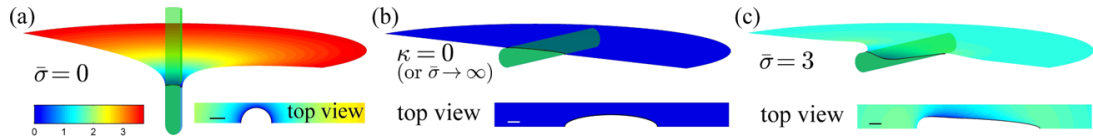

Figure 2. Simulated modes of interaction between a patch of cell membrane bounded inside a cylindrical region of radius $r_{\mathrm{m}}=20 a$ and a nanotube of radius $a$ with contact area fixed at $A_{\mathrm{c}}=10 \pi a^{2}$. (a) In the limiting case of vanishing membrane tension $\bar{\sigma}=0$, the preferred mode of interaction is the perpendicular entry mode. (b) In the limiting case of zero membrane bending stiffness $\kappa=0(\bar{\sigma} \rightarrow \infty)$, the preferred mode of interaction is a nearparallel surface adhering mode. (c) In the case of normalized membrane tension $\bar{\sigma}=3$, a nanotube interacts with the membrane at a prescribed entry angle of $\theta=15^{\circ}$. The colors represent the height of the membrane relative to the lowest point at the contact edge (black curves). Scale bars are set to be the tube radius $a$.

are scaled by the nanotube radius $a$. Other dimensionless parameters are $\bar{\gamma} \equiv 2 \gamma a^{2} / \kappa$ and $\bar{\sigma} \equiv 2 \sigma a^{2} / \kappa$.

Because the contact area $A_{c}$ is assumed to be constant, the minimum energy configuration of the nanotube-membrane system should only depend on the single dimensionless parameter $\bar{\sigma}$, which represents the ratio of membrane tension and bending energies. The bending energy plays a dominant role for $\bar{\sigma} \ll 1$, while the tension energy should do so for $\bar{\sigma} \gg$ 1. On the basis of the value of $\bar{\sigma}$, there are two limiting cases: the limit of zero-bending stiffness $(\kappa=0)$, corresponding to extremely large $\bar{\sigma} \rightarrow \infty$, and the limit of zero-tension $(\bar{\sigma}=0)$. A careful analysis of these limiting cases reveals the following two fundamental theorems regarding cell membrane interaction with nanotubes or any other relatively stiff $1 \mathrm{D}$ nanomaterials.

Theorem 1 . In the limit of vanishing membrane tension $(\bar{\sigma}=$ 0 ), a nanotube would favor the perpendicular entry mode $\theta=$ $90^{\circ}$ when interacting with an initially flat membrane at a fixed contact area. In this mode, the configuration of global minimum energy for the membrane is the catenoid

$$
x^{2}+y^{2}=a^{2} \cosh ^{2}\left(\frac{h}{a}\right), \quad z=z_{0}+h
$$

where $h$ is the height of the membrane relative to the position of the contact edge at $z=z_{0}$.

Proof. At vanishing membrane tension and fixed contact area, the elastic bending energy stored in the membrane governs the free energy of the system. The catenoid in eq 2 has unit normal vector $\mathbf{n}=\left[-a x /\left(x^{2}+y^{2}\right),-a y /\left(x^{2}+y^{2}\right),\left(1-a^{2} /\left(x^{2}+y^{2}\right)\right)^{1 / 2}\right]$. At contact edge $h=0, \mathbf{n}=(-x / a,-y / a, 0)$ satisfies the smooth contact boundary condition between the nanotube and membrane at the perpendicular entry angle of $\theta=90^{\circ}$, and $\mathbf{n}$ $\rightarrow(0,0,1)$ as $x^{2}+y^{2} \rightarrow \infty$ guarantees the asymptotic flatness of the membrane away from the contact region. The principal curvatures of the catenoid $\pm a^{-1} \operatorname{sech}^{2}(h / a)$ give rise to vanishing mean curvature $(H=0)$ everywhere in the fluid membrane, leading to zero elastic energy since $2 \kappa H^{2}=0 .{ }^{13}$ Moreover, it has been shown that the catenoid is the only complete, embedded minimal surface in the Euclidean space with zero mean curvature and two ends. ${ }^{14}$ Because the bending energy is positive definite, the catenoid in eq 2 , as shown in Figure $2 \mathrm{a}$, is the only solution of zero deformation energy for the present problem. Because of the smooth contact between the nanotube and membrane and the uniqueness of catenoid, ${ }^{14}$ any rotation of the nanotube away from the perpendicular entry configuration will cause a deviation in membrane shape from the catenoid, resulting in nonzero mean curvature and positive deformation energy. Therefore, the perpendicular entry mode $\theta$ $=90^{\circ}$ corresponds to the state of global minimum energy for a nanotube to interact with an initially flat fluid membrane at zero membrane tension. In this state, the membrane assumes the configuration of a catenoid described in eq 2 .

Theorem 2. In the limit of vanishing membrane bending stiffness $(\kappa=0)$, a nanotube interacting with an initially flat membrane under a fixed contact area would prefer an infinitesimally small entry angle $\theta \rightarrow 0^{\circ}$.

Proof. At vanishing membrane bending stiffness and fixed contact area, it becomes possible for the membrane to adopt arbitrarily sharp curvatures without any energy penalty, which effectively releases the condition of smooth contact at the contact edge. Because a flat membrane outside the nanotube automatically satisfies the condition of asymptotic flatness away from the contact region and also has smaller excess area compared to any curved surfaces, the minimum energy configuration of the membrane at any entry angle $\theta$ is just a flat surface (Figure 2b). Moreover, because the flat surface slices the nanotube along an elliptical contact edge with 

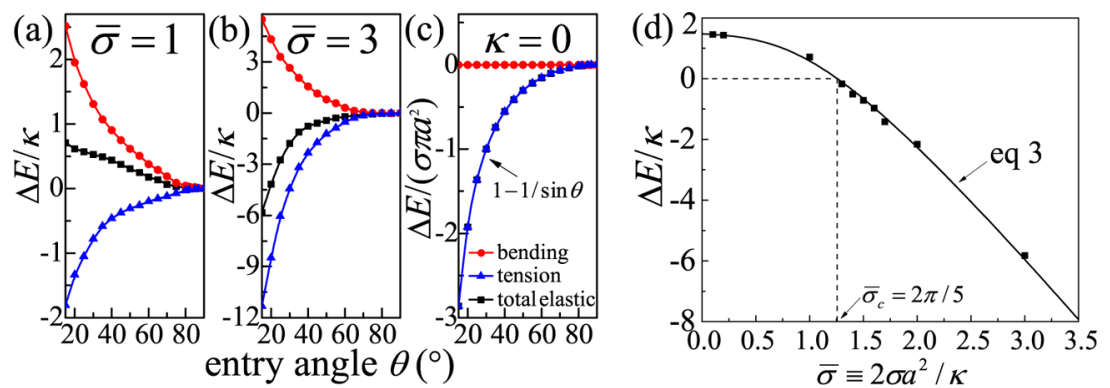

Figure 3. Variations of normalized total elastic, bending and tension energies of the membrane-nanotube system as functions of the entry angle $\theta$ for membrane with tension (a) $\bar{\sigma}=1,(\mathrm{~b}) \bar{\sigma}=3$, and (c) vanishing bending stiffness $\kappa=0$. (d) Total elastic energy change $\Delta E=E_{\mathrm{el}}\left(15^{\circ}\right)-E_{\mathrm{el}}\left(90^{\circ}\right)$ as a function of $\bar{\sigma}$. The solid squares in (d) are results from Monte Carlo simulations which are well approximated by eq 3.

semimajor axis $a / \sin \theta$ and semiminor axis $a$, resulting in an enclosed area of $\pi a^{2} / \sin \theta$, the excess membrane surface area induced by the nanotube is $\Delta A=A_{\mathrm{c}}-\pi a^{2} / \sin \theta$ and the membrane tension energy $\sigma \Delta A$ will be minimized when $\theta \rightarrow$ $0^{\circ}$. Therefore, the most favorable configuration under vanishing membrane bending stiffness and fixed contact area is for the nanotube to lie nearly parallel to the membrane, that is, $\theta \rightarrow 0^{\circ}$. In this state, the membrane wraps around the nanotube but remains a perfectly flat surface outside the contact region.

The above limiting cases define two fundamental modes of interaction between a nanotube (and any other rigid 1D cylindrical nanomaterials) and a fluid membrane. On the basis of these solutions, we postulate that a nanotube should undergo a transition from the perpendicular entry mode $\theta=90^{\circ}$ to the near-parallel mode $\theta \rightarrow 0^{\circ}$ as the normalized membrane tension $\bar{\sigma}$ increases. In the following, we confirm this transition through a rigorous numerical analysis.

In the numerical analysis, the deformed membrane configuration, as illustrated in Figure 1, is described in the Euclidean space as a tensor product cubic B-spline surface. ${ }^{15}$ The entry of the nanotube into cell at a given angle can be regarded as a steady-state process as soon as the tip region is covered by the membrane. In such a steady-state entry process limited by receptor diffusion, we can assume that the tip of the nanotube is fully wrapped and focus on energy stored in the free part of the membrane surface $S$. The position vector along $S, \mathbf{r}=\mathbf{r}(u, v)$, is parametrized by a set of curvilinear coordinates $(u, v)$ on the domain $u, v \in[0,1]$ as $\mathbf{r}(u, v)=$ $\sum_{m={ }_{0}} \sum_{n=0}^{L_{v}} \mathbf{c}_{m, n} N_{m, 4}(u) N_{n, 4}(v)$, where $\mathbf{r}(u, v)=$ $[x(u, v), y(u, v), z(u, v)]$ represents the Cartesian coordinates of the membrane and control points $\mathbf{c}_{m, n}=\left(c_{m, n}^{x}, c_{m, n}^{y}, c_{m, n}^{z}\right)$ are the coefficients of the basis functions $N_{m(n), 4}$ with integer indices 0 $\leq m \leq L_{u}$ and $0 \leq n \leq L_{v}$. The unit normal vector to $S$ is $\mathbf{n}=\left(\mathbf{r}_{u}\right.$ $\left.\times \mathbf{r}_{v}\right) /\left|\mathbf{r}_{u} \times \mathbf{r}_{v}\right|$, where $\mathbf{r}_{u} \equiv \partial \mathbf{r} / \partial u$ and $\mathbf{r}_{v} \equiv \partial \mathbf{r} / \partial v$ are two tangent vectors spanning the local tangent plane of $S$. The basis functions $N_{m, 4}(u)$ and $N_{n, 4}(v)$ can be determined explicitly by specifying two knot vectors $\left\{u_{0}, \ldots, u_{L_{u}+4}\right\}$ and $\left\{v_{0}, \ldots, v_{L_{v}+4}\right\}$, respectively. Here a typical choice is taken as $u_{i}=v_{i}=0(i=$ $0, \ldots, 3), u_{j}=1\left(j=L_{u}+1, \ldots, L_{u}+4\right)$ and $v_{k}=1\left(k=L_{v}+1, \ldots, L_{v}+\right.$ 4).

The free part of the membrane patch $S$ is bounded inside a cylindrical region of radius $r_{m}$ as shown in Figure 1. Since the system energy at entry angles $\theta$ and $\pi-\theta$ should be the same, it suffices to consider $\theta \in[0, \pi / 2]$. The inner boundary of $S$ at $u=$ 0 is constrained on the nanotube wall and described by $x^{2}+y_{0}^{2}$ $=a^{2}$ with $y_{0}=y \sin \theta+z \cos \theta$, and a smooth contact between the nanotube and membrane requires $\mathbf{n} \times \mathbf{n}_{0}=\mathbf{0}$, where $\mathbf{n}$ is the normal vector to $S$ and $\mathbf{n}_{0}=\left(x / a, y_{0} \sin \theta / a, y_{0} \cos \theta / a\right)$ is the normal vector to the nanotube wall. The outer boundary of the patch is at $u=1$ and satisfies $x^{2}+\left(y+y_{\mathrm{c}}\right)^{2}=r_{m}^{2}$ with $y_{\mathrm{c}}=$ $\left[A_{c} /(2 \pi a)-a\right] \cos \theta$ to keep the inner boundary located around the center of $S$. The membrane is assumed to be asymptotically flat with $\mathbf{n} \cdot \mathbf{n}_{1}=0$, where $\mathbf{n}_{1}=\left[x / r_{m},\left(y+y_{c}\right) /\right.$ $\left.r_{m}, 0\right]$ is the normal vector to the wall of the bounding cylinder. ${ }^{16}$ The mirror symmetry of the configuration with respect to the $y-z$ plane at $v=0,1$ requires $x(u, 0)=x(u, 1)=0$, which leads to $c_{m, 0}^{x}=c_{m, L_{v}}^{x}=0, c_{L_{w} 0}^{y}=-\left(r_{m}+y_{c}\right)$, and $c_{L_{w} L_{v}}^{y}=r_{m}-$ $y_{c}$; the condition that $\mathbf{r}_{v}$ stays parallel with $\mathbf{e}_{x}$, the base vector in the $x$-direction, leads to $c_{m, 0}^{y, z}=c_{m, 1}^{y, z}$ and $c_{m, L_{v}-1}^{y, z}=c_{m, L_{v}}^{y, z}(m=$ $\left.0, \cdots, L_{u}\right)$. The combination of conditions $\mathbf{n} \cdot \mathbf{n}_{1}=0$ at $u=1$ and $\mathbf{r}_{v} \| \mathbf{e}_{x}$ at $v=0,1$ requires $c_{L_{u}-1,0}^{z}=c_{L_{w} 0}^{z}$ and $c_{L_{u}-1, L_{v}}^{z}=c_{L_{w} L_{v}}^{z}$. To keep the height of the membrane at the outer boundary on the same level, we take $z(1,0)=z(1,1)$ which gives $c_{L_{w} 0}^{z}=c_{L_{w} L_{v}}^{z}$. To avoid penetration between the membrane and nanotube, we require $x^{2}+y_{0}^{2} \geq a^{2}$ for $u>0$.

At a given value of the normalized membrane tension $\bar{\sigma}$, the minimum energy configuration of the nanotube-membrane system is determined using a Monte Carlo simulated annealing approach. Random adjustments to the values of control points $\mathbf{c}_{m, n}$ are attempted in the Monte Carlo scheme to change the membrane shape. The patch is divided into several regions in which mesh elements have a similar size. The geometrical boundary conditions at $u=0,1$ are enforced by the least-squares method, and the membrane curvature is kept within a range. To minimize $E_{\text {tot }}$ the system temperature is gradually reduced to a value close to zero after initial equilibration. Adjustments to $\mathbf{c}_{m, n}$ are accepted according to the Metropolis algorithm. The adjustment amplitudes are controlled to make sure that about $50 \%$ adjustments are accepted on the inner and outer boundaries and in different patch regions. All simulations are performed for about $7 \times 10^{6}$ Monte Carlo moves for each $\mathbf{c}_{m, n}$ with $r_{m}=20 a, L_{u}=39$, and $L_{v}=38$. Larger membrane patch and denser mesh give similar results.

While it is well-known that punching a clamped solid membrane would result in wrinkling near the contact edge as a mechanism of releasing energy by relieving the azimuthal compression through bending, there is no wrinkling in the twodimensional fluid membrane and the energetically favorable configuration is governed by the bending and membrane tension energies (Figure 2c). As $\theta$ decreases from $90^{\circ}$, the contact edge buckles from a planar circle to a spoon-like loop (Figure 2c). This edge evolution results from a reduction in membrane tension energy, as the excess membrane area is relieved through out-of-plane displacement. 
At a fixed contact area $A_{\mathcal{c}}$, the total elastic energy $E_{\mathrm{el}} \equiv E_{\text {tot }}+$ $\gamma A_{\mathrm{c}}$ in eq 1 can be decomposed into a bending energy $E_{\mathrm{b}}$ and a tension part $\sigma \Delta A=\bar{\sigma} \Delta A \kappa /\left(2 a^{2}\right)$. Figure $3 a-c$ plots the variations of the total elastic energy change $\Delta E=E_{\mathrm{el}}(\theta)-$ $E_{\mathrm{el}}\left(90^{\circ}\right)$ and the associated bending and tension energies as functions of the entry angle $\theta$. It can be seen that the membrane bending energy tends to rotate the nanotube to the perpendicular entry angle while the membrane tension energy prefers interaction at vanishingly small entry angle (i.e., the near-parallel interaction). These behaviors are perfectly consistent with the two limiting solutions discussed earlier in the paper. The larger the membrane tension, the more likely the nanotube would stay parallel to the membrane surface. As shown in Figure 3d, an empirical relation has been found between the energy difference $\Delta E=E_{\mathrm{el}}\left(15^{\circ}\right)-E_{\mathrm{el}}\left(90^{\circ}\right)$ and $\bar{\sigma}$ as

$$
\bar{\sigma}=\bar{\sigma}_{c}+c_{2} \ln \left(1-c_{1} \frac{\Delta E}{\kappa}\right)-\frac{2 \sin \theta}{\pi(1-\sin \theta)} \frac{\Delta E}{\kappa}
$$

where $c_{1}=5 c_{2}=2 \pi^{2} / 15$ at $\theta=15^{\circ}$ and $\bar{\sigma}_{\mathrm{c}}=2 \pi / 5 \approx 1.257$ is the critical point of transition between the two interaction modes. The last linear term in eq 3 ensures $\Delta E=\sigma \pi a^{2}(1-1 / \sin \theta)$ in the limit of $\bar{\sigma} \rightarrow \infty$ as discussed in theorem 2. In the case of $\bar{\sigma}=$ $\bar{\sigma}_{\mathrm{c}}$ and $\Delta E=0$, the bending and tension energies play equal role in the regulation of nanotube rotation, and the nanotube is expected to oscillate due to thermal fluctuation.

Below the critical value $\bar{\sigma}_{c}$ the bending energy dominates over the tension energy, and the nanotube is driven to a high entry angle (the near-perpendicular entry mode); see Figures $2 \mathrm{a}$ and $3 \mathrm{a}$. The tension energy becomes dominant at large $\bar{\sigma}$, leading to a low entry angle; see Figures $2 \mathrm{c}$ and $3 \mathrm{~b}$. A comparison of Figure $3 b, c$ indicates that the tension energy variation is essentially similar to its limit behavior $-1 / \sin \theta$ once $\bar{\sigma}$ rises above its critical transition value. The competition between bending and tension energies thus leads to two different interaction modes, as found in various experiments. ${ }^{1-4}$ The profile of the total elastic energy indicates that the configurational torque $\mathrm{d} E_{\mathrm{el}} / \mathrm{d} \theta$ decreases as $\theta$ increases, and its flatness around $\theta=90^{\circ}$ suggests that the nanotube would spend much time in thermal oscillation around $90^{\circ}$ during nearperpendicular entry. In this sense, the entry angle at low $\bar{\sigma}$ may not be strictly equal to $90^{\circ}$ in experiments. This prediction is consistent with recent experimental observation that carbon nanotubes, as well as gold nanowires and asbestos nanofibers, largely adopt a near-perpendicular mode of entry into murine liver cells and human mesothelial cells. ${ }^{1}$ Similar tip entry phenomena have also been observed in successful phagocytosis of high aspect ratio filamentous Escherichia coli bacteria. ${ }^{3}$

Our analysis indicates that the cellular uptake of a 1D nanomaterial can exhibit different rotation behaviors at different normalized membrane tension $\bar{\sigma}$. For $\bar{\sigma}<\bar{\sigma}_{\mathrm{c}}$, the 1D nanomaterial would rotate to a high entry angle during uptake, and the deformed membrane forms a surface of revolution whose axis is the z-axis outside the contact region. For $\bar{\sigma}>\bar{\sigma}_{\mathrm{c}}$ the $1 \mathrm{D}$ nanomaterial would rotate to a shallow entry angle and eventually adhere to the membrane surface in a near-parallel configuration as the tip and the wall of the nanomaterial become partially expose $z$ d and partially wrapped by the weakly deformed membrane. This $\bar{\sigma}$-dependent uptake behavior should be ubiquitous in the interactions between $1 \mathrm{D}$ nanomaterials and lipid membranes. For example, recent experiments and simulations have demonstrated nanoparticles adhering to a vesicle can spontaneously form particle-chains encapsulated inside lipid tubes or linear aggregates of particles adhering on the membrane surface. ${ }^{4,17-20}$ In one experiment, transient tubular structures grow vertically on the membrane surface from adsorbed nanoparticles within substrate-supported flat giant unilamellar vesicles in an ambient solution of low $\mathrm{Ca}^{2+}$ concentrations $(1-4 \mathrm{mM}){ }^{4}$ These vertically grown tubular structures are in a tensionless state as indicated by thermal fluctuations of the membrane. As the vesicles spread on a substrate, their membrane tension increases and causes the lipid tubes to retract and the nanoparticles to align at the peripheral of the vesicle. At high concentrations of $\mathrm{Ca}^{2+}(4-10 \mathrm{mM})$, no membrane tubes form due to the adhesion-induced high membrane tension. ${ }^{4}$ Also, bacterial toxins (e.g., Shiga and cholera), when binding to glycolipid receptors on a cell, induce tubule formation at low membrane tension but fail to do so at high membrane tension. ${ }^{17}$ Numerical simulations have shown that two adsorbed nanoparticles induce a membrane tube on a vesicle at a small reduced volume but form a linear cluster on the membrane surface of a vesicle at a large reduced volume. ${ }^{20}$ This is consistent with our analysis in view of the fact that the conjugated membrane tension is greater at a larger reduced volume. On the basis of similar geometries and induced wrapping configurations, the particle-chain induced membrane tubulation and linear aggregation of nanoparticles adhering on the surface can be considered as examples analogous to the near-perpendicular tip entry at small membrane tensions and surface adhering of $1 \mathrm{D}$ nanomaterials at large membrane tensions, respectively.

Another interesting phenomenon that can be understood from the theory of membrane tension-governed nanotube rotation is that the filopodia of distinct types of cells only display a certain range of radius $(a=40-150 \mathrm{~nm}) .^{21,22}$ As finger-like structures composed of bundled actin filaments without an explicit end, the (growing) filopodia serve as an ideal biological example of $1 \mathrm{D}$ nanomaterials. To move efficiently toward a target, the filopodia involved in cell migration typically grow perpendicular to the leading edge of the cell membrane that requires $\bar{\sigma}<\bar{\sigma}_{\mathrm{c}}$ as suggested by our analysis. Considering a typical filopodial of radius $(a=40-150$ $\mathrm{nm}$ ) and membrane bending stiffness $\kappa=20 k_{\mathrm{B}} T$, the critical value $\bar{\sigma}_{\mathrm{c}} \approx 1.257$ predicted by eq 3 corresponds to membrane tension of $\sigma=0.002-0.033 \mathrm{mN} / \mathrm{m}$, which is consistent with the reported range $(0.003-0.04 \mathrm{mN} / \mathrm{m})$ for the membrane tension of neurons, ${ }^{23,24}$ a type of migrating cells displaying filopodia. This explanation also implies that the coalescence of neighboring filopodia cannot proceed constantly once the filopodial radius is above some critical size governed by the membrane tension. Microtubules in a vesicle also exhibit a similar tension-induced rotation behavior. At low membrane tension, a linear bundle of microtubules in a vesicle pushes the membrane vertically and deforms it into a tether; while at large membrane tension, the microtubules buckle into an equatorial ring attaching parallel to the membrane. ${ }^{25,26}$

In the scaling dependence of the normalized membrane tension parameter $\bar{\sigma} \equiv 2 \sigma a^{2} / \kappa$ on tube radius $a$, the $\bar{\sigma}$ dependent uptake behavior also implies a size-dependence that nanotubes with small (large) radii could rotate to configurations with high (low) entry angles. For example, while individual nanotubes could enter cells with a near-perpendicular mode, a bundle of nanotubes may lay parallel adhering to the membrane surface (e.g., Supplementary Figure S3a in ref 1). Because reported values of membrane tension vary in a wide 

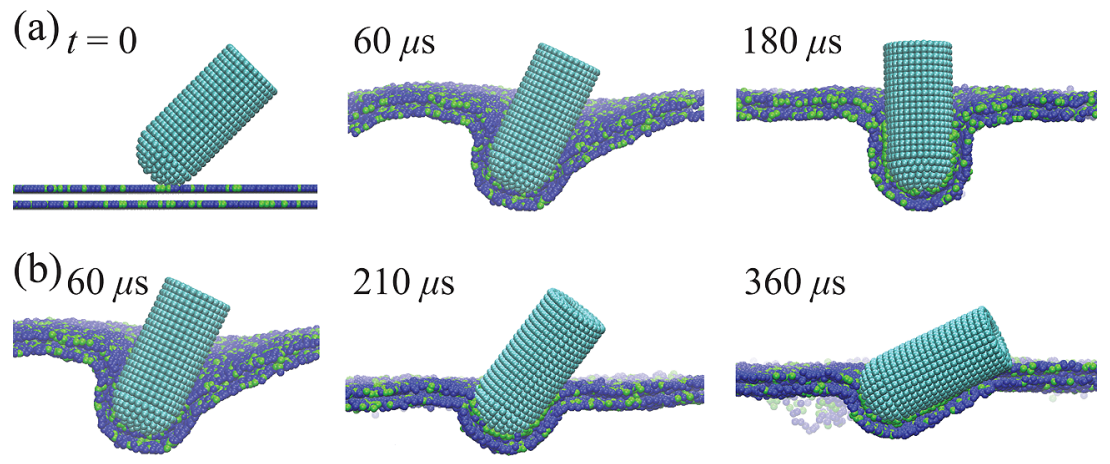

Figure 4. Time sequences of CGMD simulations showing a nanotube entering a membrane patch (blue) with an initial entry angle of $45^{\circ}$ and diffusible receptors (green) under different membrane tensions. The receptor-to-lipid ratio is $1 / 3$. The nanotube with radius of $a=10 \mathrm{~nm}$ and length of $46 \mathrm{~nm}$ is seen to rotate to (a) $90^{\circ}$ entry at a small membrane tension of $\sigma=0.03436 k_{\mathrm{B}} T / \mathrm{nm}^{2}$, corresponding to $\bar{\sigma} \equiv 2 \sigma a^{2} / \kappa=0.3436$, and (b) a near-parallel orientation at the relatively large membrane tension $\sigma=0.74 k_{\mathrm{B}} T / \mathrm{nm}^{2}$, corresponding to $\bar{\sigma}=7.4$.

range from 0.003 to $1 \mathrm{mN} / \mathrm{m}^{27}$ this size-dependent rotation behavior may provide a new and valuable complementary approach to estimate membrane tension by observing rotation behaviors of $1 \mathrm{D}$ nanomaterials with different radii. This is particularly interesting as nanophotonic tweezers based on near-field technology allow manipulating the orientation of $1 \mathrm{D}$ nanomaterials. $^{28}$

Finally, to demonstrate the role of membrane tension on the uptake pathway of 1D nanomaterials, we have also performed coarse grained molecular dynamics (CGMD) simulations by considering a multiwalled carbon nanotube binding to diffusible receptors (green) embedded in an initially flat, square patch of bilayer membrane (blue) at different membrane tensions (see Figure 4). CGMD as one of the most popular computer simulation techniques has been demonstrated to be effective and efficient in studying biomembrane $e^{1,29-39}$ and other biological systems. ${ }^{40,41}$ Because the time scale associated with the equilibration of deformed lipid membranes is much smaller than the typical time for dynamic cellular uptake mediated by receptor diffusion, we focus on the shorter time scale and assume in the preceding theoretical analysis that the area of the contact region remains constant as the $1 \mathrm{D}$ nanomaterial adjust its entry angle to reduce the elastic energy of the membrane. That particular assumption is relaxed in the following CGMD simulations whose results further support its validity. The simulation setup here is similar to that used in our earlier study, ${ }^{1}$ where each lipid and receptor molecule is modeled as three connected beads with one hydrophilic head-bead and two hydrophobic tail-beads. The bead diameter, $d_{\mathrm{b}}$, is set to $1 \mathrm{~nm}$ to construct a bilayer with an appropriate thickness. The CGMD simulations are first performed at constant temperature $\left(k_{\mathrm{B}} T=\right.$ $1.1 \varepsilon$ ) and pressure with a dissipative particle dynamics (DPD) thermostat. A damping parameter $\Gamma=1.0 \varepsilon \tau / d_{\mathrm{b}}^{2}$ with $\tau=15 \mathrm{~ns}$ and a cutoff distance $d_{\text {cut }}=3.0 d_{\mathrm{b}}$ are used in the thermostat. In Figure $4 \mathrm{a}$, the projected area of the membrane shrinks from 100 $\times 100 \mathrm{~nm}^{2}$ at $t=0$ to $92 \times 92 \mathrm{~nm}^{2}$ at $t=60 \mu \mathrm{s}$ as the membrane wraps around the nanotube. The lateral dimension of the bilayer is then fixed and the simulation system is adjusted to be at constant temperature and volume conditions. For a membrane with a fixed lateral dimension, the membrane tension can be effectively controlled by randomly deleting a certain amount of lipid molecules. Such a procedure, often referred to as the N-varied DPD method, has been widely used to investigate the interaction between nanoparticles and cell membrane. ${ }^{33-37}$ In Figure 4a, no lipids has been deleted, which yields a membrane tension of $\sigma=0.0378 \varepsilon / d_{\mathrm{b}}^{2}=0.03436 k_{\mathrm{B}} T /$ $\mathrm{nm}^{2}$ or $\bar{\sigma} \equiv 2 \sigma a^{2} / \kappa=0.3436$ with $\kappa=20 k_{\mathrm{B}} T$, and the system equilibrates at about $t=180 \mu \mathrm{s}$ with an entry angle around $90^{\circ}$. In contrast, in Figure $4 \mathrm{~b}$ we simulate a larger membrane tension by adopting a similar procedure as that in Figure $4 a$, except that $13 \%$ lipid molecules are deleted from the membrane at $t=60$ $\mu \mathrm{s}$. In this case, the membrane tension is $\sigma=0.814 \varepsilon / d_{\mathrm{b}}^{2}$ (or $\bar{\sigma}=$ 7.4 with $\kappa=20 k_{\mathrm{B}} T$ ) and the nanotube rotates to a lower entry angle. These simulations clearly demonstrate the essential physics revealed by our theoretical analysis that the nanotube would prefer a low entry angle at a large tension and nearperpendicular entry at a small $\bar{\sigma}$.

A related but different phenomenon is the orientational changes of a short nanorod wrapped around by a membrane that would occur in the middle stage of the wrapping process no matter how large or small the membrane tension is. Recent studies showed that short rigid adhesive nanorods including ellipsoidal nanoparticles with small aspect ratios orient their longer axes parallel to the membrane first and remain in that configuration until about a half of the particle surface area is wrapped, and then undergo a configurational transition by realigning their longer axes perpendicular to the membrane during further wrapped. ${ }^{38,39,42,43}$ After the transition moment, a short nanorod remaining parallel to the membrane with both highly curved ends covered by the membrane would cost more membrane bending energy than that the perpendicular configuration in which only one end needs to be covered by the membrane. Here, the membrane bending energy difference results in nanoparticle reorientation. This reorientation of short rigid nanorods caused by membrane wrapping has also been observed in cellular uptake of very soft nanoparticles in which the soft particle adheres on the membrane with its flattened surface at early stage of wrapping but then changes its configuration to one with longer axis perpendicular to the membrane during the late stage of wrapping. ${ }^{44}$ Compared with our present analysis on tension-controlled two modes of interaction between 1D nanomaterials and lipid membranes, the mode of interaction between short nanorods and lipid membranes consists of two stages distinguished by a particle reorientation regardless of the value of membrane tension.

The cellular uptake of nanomaterials can usually be considered as a process limited by diffusion of receptors in the cell membrane toward the contact region., ${ }^{1,11}$ For the uptake of cylindrical nanoparticles staying parallel to the membrane, the wrapping process is controlled by the onedimensional diffusion of receptors and the wrapped area should scale as $\sim \alpha_{1 \mathrm{~d}} L(D t)^{1 / 2}$ where $D$ is the diffusivity of receptors in 
the membrane, $t$ is the wrapping time, $L$ is the length of the cylindrical particle, and $\alpha_{1 \mathrm{~d}}$ is a dimensionless constant, commonly referred to as the speed factor, which depends on the initial receptor density $\xi_{0}$ before contact with nanoparticles, the receptor-ligand bond density $\xi_{\mathrm{L}}$ in the contact region, the binding energy of a receptor-ligand bond $e_{\mathrm{RL}} k_{\mathrm{B}} T$, and the elastic energy of the membrane. ${ }^{11}$ In contrast, the wrapping process of sphere-like nanoparticles involves two-dimensional diffusion of receptors and the wrapped area should scale as $\sim$ $\alpha_{2 \mathrm{~d}}^{2} D t$, where $\alpha_{2 \mathrm{~d}}$ is the corresponding speed factor. Taking typical parameter values as $e_{\mathrm{RL}}=15, \xi_{\mathrm{L}}=5 \times 10^{3} \mu \mathrm{m}^{-2}, r=20$ $\mathrm{nm}, \kappa=20 k_{\mathrm{B}} T$ and $\xi_{0} / \xi_{\mathrm{L}}=0.1$, we estimate $\alpha_{1 \mathrm{~d}} \approx 0.0603$ and $\alpha_{2 \mathrm{~d}} \approx 0.185$ from our previous study, ${ }^{11}$ meaning that membrane wrapping regulated by two-dimensional diffusion of receptors is much faster than that regulated by one-dimensional receptor diffusion. For 1D nanomaterials, membrane wrapping of their curved tip should be governed by two-dimensional diffusion of receptors, while wrapping of their cylindrical wall is regulated by one-dimensional diffusion of receptors. This means that the tip, rather than the lateral wall, of a $1 \mathrm{D}$ nanomaterial should be wrapped first, and the subsequent interaction is governed by the two interaction modes controlled by the normalized membrane tension $\bar{\sigma} \equiv 2 \sigma a^{2} / \kappa$, as shown in our present study. For short nanorods with two curved tips and a much smaller lateral wall, the difference between receptor diffusion between the tip and wall regions may not be evident. Therefore, the initial wrapping of short nanorods would be mainly governed by the elastic energy of the deformed membrane. Such nanorods would align with their long axes parallel to the membrane first and reorient perpendicular to the membrane during the late stage of wrapping regardless of the value of membrane tension. As the length of the nanorod increases, the receptor diffusion in the early stage of wrapping and membrane tension become more important and begin to dominate the wrapping process beyond a critical length. A detailed study on such length effect of rod-shaped nanoparticles on cellular uptake is out of the scope of the current study and deserves further investigations.

Some exceptional cases that are somewhat related to the present study are worth mentioning. First, in a theoretical limiting but physiologically irrelevant case in which the cell membrane is fully covered with receptors, the interaction between the membrane and adhesive nanomaterials becomes nonspecific and a 1D nanomaterial would adhere parallel to the membrane, as demonstrated in our previous study (see Figure $3 c$ in ref 1). Second, we have focused on clathrin-free endocytosis where the membrane deformation serves as the dominant resistance to nanoparticle entry. In clathrin-mediated endocytosis, the cell membrane and the underlying cortical actin cytoskeleton form a composite physical barrier to the entry of nanomaterials, which would involve both membrane deformation and remodeling of actin cytoskeleton. ${ }^{45-48}$ Recent theoretical study indicates that the relative contribution of membrane deformation and cytoskeleton remodeling to the entry resistance depends nonlinearly on the size of nanomaterials, the elastic and viscous properties of actin cytoskeleton, and the stage of endocytosis. ${ }^{48}$ A thorough understanding of these effects in cellular uptake would call for more sophisticated models.

In summary, we have performed both theoretical analysis and molecular dynamics simulations to study the cellular uptake of one-dimensional nanomaterials such as nanotubes, nanowires, nanofibers, and certain nanoparticle-chains. First, we have established exact solutions to the problem in the limiting cases of vanishing bending stiffness or vanishing membrane tension, revealing two fundamental modes of interaction between onedimensional nanomaterials and lipid membranes: a perpendicular entry mode and a parallel adhering mode. On the basis of the limiting solutions, we postulated that there exists a transition between these two modes as the normalized membrane tension parameter $\bar{\sigma} \equiv 2 \sigma a^{2} / \kappa$ increases. This hypothesis is then confirmed through a numerical analysis based on a Monte Carlo method. In the numerical analysis, we have calculated the variations of the system energy as a nanotube is wrapped into a patch of membrane at a prescribed entry angle. We conclude that perpendicular tip entry and parallel surface adhering are two basic modes of cell interaction with one-dimensional nanomaterials, controlled by a single dimensionless parameter, the normalized membrane tension $\bar{\sigma}$ $\equiv 2 \sigma a^{2} / \kappa$ that depends on the nanomaterial radius $a$, membrane tension $\sigma$, and bending stiffness $\kappa$ of the membrane. This $\bar{\sigma}$-dependent uptake behavior is ubiquitous in the interplay between cell membranes and one-dimensional nanomaterials. The B-spline based simulation method for evolving lipid membrane surfaces used in the present analysis can be extended to membrane wrapping around nanomaterials of more complex geometries, such as ellipsoidal nanoparticles and cylinders with elliptic cross sections. Our results can also serve as a foundation for future studies taking into account membrane fluctuation, ${ }^{49,50}$ receptor diffusion, ${ }^{11,51}$ receptor-ligand binding, ${ }^{52}$ deformation of cytoskeleton, ${ }^{48}$ elasticity of nanomaterials, ${ }^{44}$ and simultaneous interaction with multiple nanoparticles. ${ }^{16,18-20,31,53,54}$

\section{AUTHOR INFORMATION}

\section{Corresponding Author}

*E-mail: huajian_gao@brown.edu.

\section{Notes}

The authors declare no competing financial interest.

\section{ACKNOWLEDGMENTS}

X.Y. and H.G. acknowledge support by the National Science Foundation under INSPIRE: "Computational Design for the Safe Development of High-Aspect-Ratio Nanomaterials”, CBET-1344097, and one of its predecessor grants, CMMI1028530; X.S. acknowledges support by the National Natural Science Foundation of China (Grant 11272327 and 11023001).

\section{REFERENCES}

(1) Shi, X.; von dem Bussche, A.; Hurt, R. H.; Kane, A. B.; Gao, H. Nat. Nanotechnol. 2011, 6, 714-719.

(2) Sanchez, V. C.; Weston, P.; Yan, A.; Hurt, R. H.; Kane, A. B. Part. Fibre Toxicol. 2011, 8, 17.

(3) Möller, J.; Luehmann, T.; Hall, H.; Vogel, V. Nano Lett. 2012, 12, 2901-2905.

(4) Gözen, I.; Billerit, C.; Dommersnes, P.; Jesorka, A.; Orwar, O. Soft Matter 2011, 7, 9706-9713.

(5) Sun, M.; Graham, J. S.; Hegedüs, B.; Marga, F.; Zhang, Y.; Forgacs, G.; Grandbois, M. Biophys. J. 2005, 89, 4320-4329.

(6) Haluska, C. K.; Riske, K.; Marchi-Artzner, V.; Lehn, L.-M.; Lipowsky, R.; Dimova, R. Proc. Natl. Acad. Sci. U.S.A. 2006, 103, 15841-15846.

(7) Nam, J.; Santore, M. M. Phys. Rev. Lett. 2011, 107, 078101.

(8) Panyam, J.; Labhasetwar, V. Pharm. Res. 2003, 20, 212-220.

(9) Gauthier, N. C.; Monzo, P.; Kaddai, V.; Doye, A.; Ricci, V.; Boquet, P. Mol. Biol. Cell 2005, 16, 4822-4866. 
(10) Chithrani, B. D.; Chan, W. C. W. Nano Lett. 2007, 7, 1542(54) Šarić, A.; Cacciuto, A. Soft Matter 2013, 9, 6677-6695. 1550

(11) Gao, H.; Shi, W.; Freund, L. B. Proc. Natl. Acad. Sci. U.S.A. 2005, 102, 9469-9474.

(12) Helfrich, W. Z. Naturforsch. C 1973, 28, 693-703.

(13) Deserno, M. Phys. Rev. E 2004, 69, 031903.

(14) Schoen, R. M. J. Differ. Geom. 1983, 18, 791-809.

(15) Farin, G. Curves and Surfaces for CAGD: A Practical Guide, 5th ed.; Morgan Kaufmann: San Francisco, 2002.

(16) Reynwar, B. J.; Deserno, M. Soft Matter 2011, 7, 8567-8575.

(17) Ewers, H.; et al. Nat. Cell Biol. 2010, 12, 11-18.

(18) Šarić, A.; Cacciuto, A. Phys. Rev. Lett. 2012, 108, 118101.

(19) Šarić, A.; Cacciuto, A. Phys. Rev. Lett. 2012, 109, 188101.

(20) Bahrami, A. H.; Lipowsky, R.; Weikl, T. R. Phys. Rev. Lett. 2012, 109, 188102.

(21) Medalia, O.; Beck, M.; Ecke, M.; Weber, I.; Neujahr, R.; Baumeister, W.; Gerisch, G. Curr. Biol. 2007, 17, 79-84.

(22) Mattila, P. K.; Lappalainen, P. Nat. Rev. Mol. Cell Bio. 2008, 9, $446-454$.

(23) Hochmuth, F. M.; Shao, J.-Y.; Dai, J.; Sheetz, M. P. Biophys. J. 1996, 70, 358-369.

(24) Dai, J.; Sheetz, M. P.; Wan, X.; Morris, C. E. J. Neurosci. 1998, $18,6681-6692$.

(25) Fygenson, D. K.; Marko, J. F.; Libchaber, A. Phys. Rev. Lett. 1997, 79, 4497-4500.

(26) Fygenson, D. K.; Elbaum, M.; Shraiman, B.; Libchaber, A. Phys. Rev. E 1997, 55, 850-859.

(27) Morris, C. E.; Homann, U. J. Membr. Biol. 2001, 179, 79-102.

(28) Kang, P.; Serey, X.; Chen, Y.-F.; Erickson, D. Nano Lett. 2012, $12,6400-6407$.

(29) Marrink, S. J.; de Vries, A. H.; Mark, A. E. J. Phys. Chem. B 2004, $108,750-760$.

(30) Limbach, H. J.; Arnold, A.; Mann, B. A.; Holm, C. Comput. Phys. Commun. 2006, 174, 704-727.

(31) Reynwar, B. J.; Illya, G.; Harmandaris, V. A.; Müller, M. M.; Kremer, K; Deserno, M. Nature 2007, 447, 461-464.

(32) Yang, K.; Ma, Y.-Q. Nat. Nanotechnol. 2010, 5, 579-583.

(33) Hong, B.; Qiu, F.; Zhang, H.; Yang, Y. J. Phys. Chem. B 2007, 111, 5837-5849.

(34) Yue, T.; Li, S.; Zhang, X.; Wang, W. Soft Matter 2010, 6, 61096118.

(35) Li, Y.; Yue, T.; Yang, K.; Zhang, X. Biomaterials 2012, 33, 49654973.

(36) Yue, T.; Zhang, X. ACS Nano 2012, 6, 3196-3205.

(37) Yue, T.; Zhang, X. Soft Matter 2013, 9, 559-569.

(38) Vácha, R.; Martinez-Veracoechea, F. J.; Frenkel, D. Nano Lett. 2011, 11, 5391-5395.

(39) Huang, C.; Zhang, Y.; Yuan, H.; Gao, H.; Zhang, S. Nano Lett. 2013, 13, 4546-4550.

(40) Buehler, M. J.; Yung, Y. C. Nat. Mater. 2009, 8, 175-188.

(41) Nova, A.; Keten, S.; Pugno, N. M.; Redaelli, A.; Buehler, M. J. Nano Lett. 2010, 10, 2626-2634.

(42) Dasgupta, S.; Auth, T.; Gompper, G. Soft Matter 2013, 9, 54735482 .

(43) Bahrami, A. H. Soft Matter 2013, 9, 8642-8646.

(44) Yi, X.; Shi, X.; Gao, H. Phys. Rev. Lett. 2011, 107, 098101.

(45) Coller, K. E.; Berger, K. L.; Heaton, N. S.; Cooper, J. D.; Yoon, R.; Randall, G. PLoS Pathog. 2009, 5, e1000702.

(46) Stolp, B.; Fackler, O. T. Viruses 2011, 3, 293-311.

(47) Taylor, M. P.; Koyuncu, O. O.; Enquist, L. W. Nat. Rev. Microbiol. 2011, 9, 427-439.

(48) Li, L.; Liu, X.; Zhou, Y.; Wang, J. Biophys. J. 2012, 102, 22302233.

(49) Golestanian, R.; Goulian, M.; Kardar, M. Phys. Rev. E 1996, 54, 6725-6734.

(50) Weikl, T. R. Europhys. Lett. 2001, 54, 547-553.

(51) Freund, L. B.; Lin, Y. J. Mech. Phys. Solids 2004, 52, 2455-2472.

(52) Lin, Y.; Freund, L. B. Phys. Rev. E 2008, 78, 021909.

(53) Weikl, T. R. Eur. Phys. J. E 2003, 12, 265-273. 